\title{
Warping two-dimensional electrophoresis gel images to correct for geometric distortions of the spot pattern
}

\author{
John S. Gustafsson*广
}

\author{
Anders Blomberg
}

Mats Rudemo ${ }^{\dagger}$

November 26, 2001

\begin{abstract}
A crucial step in two-dimensional gel based protein expression analysis is to match spots in different gel images that correspond to the same protein. It still requires extensive and time-consuming manual interference, although several semiautomatic techniques exist. Geometric distortions of the protein patterns inherent to the electrophoresis procedure are one of the main causes of these difficulties. An image warping method to reduce this problem is presented. A warping is a function that deforms images by mapping between image domains. The method proceeds in two steps. Firstly, a simple physicochemical model is formulated and applied for warping of each gel image to correct for what might be one of the main causes of the distortions: current leakage across the sides during the second dimension electrophoresis. Secondly, the images are automatically aligned by maximizing a penalized likelihood criterion. The method is applied to a set of ten gel images showing the radioactively labeled proteome of yeast Saccharomyces cerevisiae during normal and steady-state saline growth. The improvement in matching when given the warped images instead of the original ones is exemplified in a comparison within a commercial software.
\end{abstract}

Keywords: Alignment; Curved field lines; Image analysis; Proteomics;

Two-dimensional gel electrophoresis

\section{Introduction}

Two-dimensional (2D) gel electrophoresis is still the major technique in quantitative global protein expression analysis. A crucial step in this technique is image analysis where the

* Correspondence: John S. Gustafsson, Department of Mathematical Statistics, Chalmers University of Technology, SE-412 96 Göteborg, Sweden. E-mail: johng@math.chalmers.se Fax: +46-31-7723508

${ }^{\dagger}$ Department of Mathematical Statistics, Chalmers University of Technology

$\ddagger$ Department of Cell and Molecular Biology - Microbiology, Göteborg University 
protein spot patterns in different images are to be matched. Two main steps are common in the current methodology of 2D gel image analysis: an initial spot detection and quantification step, and a subsequent matching step (Pleissner et al., 2001). Both these steps are non-trivial and often need time-consuming manual interference. The matching step is a bottleneck in the gel analysis which has consequences for a comprehensive statistical analysis (Voss and Haberl, 2000), especially in large-scale proteomics. One main cause that pushes the semiautomatic matching algorithms to their limits is geometric distortions of the protein patterns inherent to the casting, polymerization and running procedure of the gels.

Image warping is a function which deforms images; all positions in one image plane are mapped to positions in another plane. It has many applications in the field of image analysis (Glasbey and Mardia, 1998): for example to remove optical distortions caused by camera lenses or viewing perspective, to register an image with a template, or to bring two or more images into alignment. In the registration and alignment cases, the choice of warp is a compromise between achieving a good match and introducing a smooth deformation. In the analysis of 2D electrophoresis gel images, the warping technique has mainly been used to align images for visual comparison of gels (Horgan et al., 1992; Appel et al., 1997; Lemkin, 1997; Pleissner et al., 1997). In these examples the smoothness of the warp is ensured with the usage of smooth functions, such as low-degree polynomials or thin-plate splines, and the function is specified to fit a set of manually added landmarks. Another example related to 2D gels is a warping method to remove geometric distortions, so called "smiles" and "frowns", in multi-track electrophoresis gels (Glasbey and Wright, 1994).

Image warping can also be used for automatic alignment of $2 \mathrm{D}$ gel images. In the common spot matching approach the data in the images are initially reduced to lists of spot features in the detection step, and one of these features, the spot center, is used in the following matching step. With a pixel-based alignment method one is able to use all data in the gel, such as spot shape, streaks and reproducible background features. In an early example of this warping approach Conradsen and Pedersen (1992) apply linear transformation at a progressively finer series of scales, to minimize the sum of squared pixel-by-pixel differences. However, no smoothness constraints are considered. A recent contribution to the methodologies in $2 \mathrm{D}$ gel analysis that uses the warping technique for image alignment is the one presented by Smilansky (2001). A sequence of covering subrectangles in the gel image is constructed such that each sub-rectangle contains a small number of spots. A pixel-based alignment is then applied for each sub-rectangle, and the shift vectors of these sub-image alignments are combined into a global warping using Delaunay triangulation. The similarity measure in the sub-image alignment is made up by several components based on both contrast and gradient information in the images. Too rough distortions are avoided by restrictions when changing the shift vectors in the iteration loop.

We here present another warping method to correct for smooth geometric distortions of the spot patterns in $2 \mathrm{D}$ gels, which applied prior to the common matching procedure should reduce problems due to these distortions. The method proceeds in two steps.

Firstly, a warping of each gel image is applied to correct for what might be one main 
cause of distortion: current leakage along the sides of the gel in the second dimension gel electrophoresis. The effect of current leakage on the electric field in the gel and its consequences for the protein pattern is investigated with a simple physicochemical model. In the case of multi-track gels, it is possible to use the fact that bands are asymmetric to estimate orientation directions, and then to formulate a mathematical model of the warping function (Glasbey and Wright, 1994). It is harder to extract similar directional information in 2D gels. An easily identifiable object with a direction in a 2D gel is the gel front at the bottom of the gel. In the presence of current leakage, the gel front is deformed from a straight line. We use the curve form of the gel front to estimate the amount of current leakage.

Secondly, the current leakage corrected images are automatically aligned to correct for other sources of geometric distortions. There are many approaches to image alignment (Glasbey and Mardia, 1998). We have adopted a statistical formulation proposed by Glasbey and Mardia (2001) where the compromise between achieving a good match and introducing rough distortions is formulated as to maximize a penalized likelihood. The likelihood measures the similarity between aligned images and the penalty is a distortion criterion, uniquely minimized by a null-set of functions. We use a simple Gaussian model for the warped image after initial logarithmic intensity transformations of the images, and as distortion criterion we choose one that as null-set has the set of affine functions. Within this unifying statistical framework one has the flexibility to consider other image models and null-sets in the future.

The method is applied to a set of ten 2D polyacrylamide gel electrophoresis (2DPAGE) images from an analysis of protein expression changes in the yeast Saccharomyces cerevisiae during steady-state saline growth (Alipour et al., 2001). The protein pattern has already been manually matched in this analysis and the list of matched spot centers serves as reference in a geometric similarity measure of the protein patterns in the gel images. Finally, the improvement in matching when given the warped images instead of the original ones is exemplified in a comparison within a commercially available software.

\section{Material and methods}

A warping function is a function that maps positions $x=\left(x_{1}, x_{2}\right)$ in one image domain to positions $f(x)=\left(f_{1}(x), f_{2}(x)\right)$ in another image domain. Let $Y$ be an image and let $Y_{x}$ denote the pixel value at position $x$. The warped version of $Y$ under $f$, denoted $Y_{f}$, is specified by

$$
\left(Y_{f}\right)_{x}=Y_{f(x)}, \quad \text { for } x \in X,
$$

where $X=\left\{1, \ldots, n_{1}\right\} \times\left\{1, \ldots, n_{2}\right\}$ is the domain of $Y_{f}$. This means that the pixel value at position $x$ in the warped image $Y_{f}$ is defined to be the value at position $f(x)$ in the original image $Y$. If $f_{1}(x)$ and $f_{2}(x)$ are not integers we use bilinear interpolation to calculate the pixel value and if $f(x)$ lies outside the domain of $Y$ we define $Y_{f(x)}$ to be a constant value. 
Ideally this constant value would be zero, no protein contents, but we use an estimate of the background level defined as the median value of the right and left-most edges of $Y$.

\subsection{Gel images}

The ten gel images are from an analysis of protein expression changes in the yeast Saccharomyces cerevisiae during steady-state saline growth (1M NaCl) (Alipour et al., 2001). Normal and saline growth conditions were replicated in five individual samples. The proteins were radioactively labeled with ${ }^{35} \mathrm{~S}$-methionine during growth. Protein samples were extracted from harvested cells and $2 \cdot 10^{6}$ DPM applied per gel.

The 2D-PAGE was run on an Investigator ${ }^{\mathrm{TM}}$ system (Oxford Glycosystem Ltd) as described in (Norbeck and Blomberg, 1997). In brief, each protein sample was subjected to isoelectric focusing in a gel strip containing an immobilized $\mathrm{pH}$ gradient ( $\mathrm{pH} 3-10)$. The second dimension polyacrylamide gels were cast between two glass plates separated from each other by two thin plastic spacers glued between the plates along each vertical side. The width of the plastic spacers was $\Delta w=25 \mathrm{~mm}$, and the gel width and height were $w=235 \mathrm{~mm}$ and $h=215 \mathrm{~mm}$. The first dimension strips were equilibrated in a sodium dodecyl sulfate (SDS) buffer and mounted on the SDS-PAGE slab gels. The second dimension gel electrophoresis was run on a vertical system at a limiting power of $16 \mathrm{GW}$ (maximum voltage $500 \mathrm{~V}$ ) and with gels fully submerged in running buffer for efficient cooling. Immediately after the run the gels were dried on filter paper.

Dried gels were exposed to phosphor image plates, which were scanned in a PhosphorImager $^{\mathrm{TM}}$ (Molecular Dynamics Inc.) with a pixel size of $176 \times 176 \mu \mathrm{m}$ and stored as 16bits TIFF-images. The images were processed as described in (Blomberg et al., 1995) and 1028 spots were manually matched in all ten gel images. The gels were numbered such that gels 1-5 were from normal growth conditions and gels 6-10 were from steady state saline growth.

\subsection{Warping step I: Current leakage correction}

Despite the wide application of SDS-PAGE considerable uncertainty still exists about the migration behavior of the SDS-protein complexes (Westerhuis et al., 2000; Kozulic, 1995). In an analysis of electrophoretic transport in porous media, of which SDS-PAGE is an example, four major factors have to be considered: (i) the structure of the media, the polyacrylamide net; (ii) the characteristics of the transporting solute, the SDS-protein complexes; (iii) the solvent conditions, the buffer; and finally (iv) the nature of the electric field. The role of the gel and the size and shape of the SDS-protein complexes are the factors considered in the two dominant models of gel migration of SDS-protein complexes; the standard Ogston-Morris-Rodbard-Chrambach model (Rodbard and Chrambach, 1970) and the reptation model (Guo and Chen, 1990) - reptation as in reptile-like motion. These two factors are also the ones investigated in a recent contribution to the general gel electrophoresis theory (Boileau and Slater, 2001). Buffer conditions in dif- 
ferent electrophoresis techniques are modeled in (Bier et al., 1983). The effect of the geometry and electric conductivity of gel fibers on the local electric field and its consequences for the effective mobility has recently been considered (Locke and Trinh, 1999; Mercier et al., 2001).

The major factor in the geometric distortions of the protein pattern due to current leakage is a global change of the electric field. Therefore, we focus on the global electric field in our model and a simple approach is applied to the other three of the four factors listed above. The experimental setups considered are gels that are not perfectly isolated along the sides, and the electric potential outside this incomplete isolation is the same as at the anode. This situation arises in gels, where there may be imperfections in the gluing of the spacers and the gels are run fully submerged in running buffer.

\subsubsection{Current leakage model}

General assumptions The electrostatic potential in the gel, $\Psi$ [V], satisfies Poisson's equation

$$
-\nabla \cdot(\epsilon \nabla \Psi)=\rho,
$$

where $\nabla[1 / \mathrm{m}]$ is the gradient operator, $\rho\left[\mathrm{C} / \mathrm{m}^{3}\right]$ is the net charge volume density and $\epsilon$ $[\mathrm{As} / \mathrm{Vm}]$ is the permittivity of the medium. In general, the species continuity equations for all charged species in the solute and solvent have to be considered and coupled to Eq. (2), but when the concentration of the solute is much lower than that of the current carrying ions in the solvent, electro-neutrality, that is $\rho=0$, can be assumed and a decoupling of the electric potential from the migration of the solute and solvent is possible (Locke, 1998). The media and solvent is looked upon as a single homogeneous unit, referred to as the gel. The migration velocity, $v[\mathrm{~m} / \mathrm{s}]$, of the SDS-protein complexes in the gel is assumed to be a linear function of the electric field, the negative gradient of the electric potential:

$$
v=-\nu \nabla \Psi,
$$

where $\nu\left[\mathrm{m}^{2} / \mathrm{Vs}\right]$ is the mobility coefficient of the SDS-protein complex. We assume that the mobility coefficient is spatially uniform and independent of the electric field strength.

The spatial dimensions are denoted in terms of a gel run on a vertical system. The geometry can be seen in Fig. 1, where the cathode and anode are the top and bottom boundaries, respectively. The first dimension iso-electric focused proteins are put on top and migrate downwards in the second dimension gel electrophoresis. The upper left corner of the gel is defined as the origin, with the horizontal dimension denoted $x_{1}$ and the vertical dimension $x_{2}$. Any dependency of the third dimension is disregarded, and all spatial quantities are considered to be two-dimensional. 
Electrostatic potential in gel With assumptions of electro-neutrality and a uniform permittivity, the electrostatic potential in the gel is given by Laplace's equation

$$
\nabla^{2} \Psi=0,
$$

with boundary conditions

$$
\begin{aligned}
\Psi & =0 & & \text { at the anode (bottom), } \\
\frac{\partial \Psi}{\partial n}+\gamma_{l} \Psi & =0 & & \text { at the left boundary, } \\
\Psi & =-V_{0} & & \text { at the cathode (top) } \\
\frac{\partial \Psi}{\partial n}+\gamma_{r} \Psi & =0 & & \text { at the right boundary. }
\end{aligned}
$$

Here $V_{0}[\mathrm{~V}]$ denotes the absolute value of the applied voltage and $\partial \Psi / \partial n$ is the outward normal derivative of $\Psi$ at the boundary.

At the anode and cathode boundaries $\Psi$ is constrained by Dirichlet conditions. The generalized Neumann condition at the two vertical boundaries is derived from Ohm's law and the law of charge conservation. In brief, the first term in the condition originates from the component of the current field in the gel that is orthogonal to the boundary, and the second term originates from an expression for the leakage current in the imperfect gluing of the spacer, where it is assumed that the spacer width $\Delta w$ is small in comparison with the spacer length. The quantity that determines the amount of current leakage is

$$
\gamma=\frac{\sigma_{\text {side }}}{\sigma_{\text {gel }} \Delta w},
$$

where $\sigma_{g e l}$ and $\sigma_{\text {side }}[\mathrm{A} / \mathrm{V}]$ are the conductivities in the gel and in the gluing of the spacer, respectively. The larger $\gamma$ is, the larger is the current leakage. Note that $\gamma_{l}$ and $\gamma_{r}$ are functions of the second dimension coordinate along the boundaries.

Warping of the protein pattern The geometric distortions of the protein pattern due to current leakage is a function that maps the position $x$ that a protein would have had in an ideal gel with perfect isolation along the sides to the corresponding position $f(x)$ in a gel with leakage. This current leakage warping function $f(x)$ is given by a set of ordinary differential equations in $x_{2}$, one for each $x_{1}$. Each differential equation determines the map of positions along a migration path in the ideal gel to positions along the corresponding path in the gel with current leakage:

$$
\frac{\partial}{\partial x_{2}} f(x)=-\frac{h}{V_{0}} \nabla \Psi(f(x)), \text { for } x_{2} \in(0, c),
$$

with initial value

$$
f\left(\left(x_{1}, 0\right)\right)=\left(x_{1}, 0\right), \quad \text { for } x_{1} \in(a, b) .
$$


Here $a[\mathrm{~m}]$ and $b[\mathrm{~m}]$ are the left-most and right-most $x_{1}$-coordinate of the first dimension strip, and $c[\mathrm{~m}]$ denotes the $x_{2}$-position of the gel front in the ideal gel; the gel front consists of proteins and protein fragments that are too small to be resolved in the gel. Hence, these numbers delimit the area that contains proteins in the ideal gel: $(a, b) \times(0, c)$.

The differential equation is derived by considering the velocity equation (3) for a protein in both gels, noticing that time can be expressed in terms of settled distance in the second dimension in the ideal gel and using this substitution in the differential equation for the migration in the gel with leakage. Note that the mobility coefficient has canceled out in Eq. (10).

Governing dimensionless quantities By putting the model equations on dimensionless form it is possible to identify dimensionless combinations of the physical quantities that alone govern the result. A dimensionless formulation also provides proper scaling of the quantities for numerical treatment. A natural choice is to express all length quantities in terms of the gel height $h$ and the electric potential in terms of the absolute value of the applied voltage, $V_{0}$.

There are two governing dimensionless combinations of the physical quantities: the ratio of the gel width and the gel height, $w / h$, and a dimensionless version of the conductivity expression in Eq. (9), $h \gamma$. Hence, the scaled current leakage warping function is fixed by specifying the geometry ratio and the dimensionless conductivities along the two vertical sides with two arbitrary functions.

\subsubsection{Parameter estimation}

To be able to use the current leakage model one has to consider two tasks. Firstly, one has to know the map between the gel image domain and the model gel domain. Secondly, one has to estimate the governing dimensionless quantities in the model to calculate a current leakage warping function. In order to solve these tasks, we extract two features in the gel image: a straight line is fitted to the top edge and a low-degree polynomial curve is fitted to the gel front. We have done this manually by specifying two points on the cathode gel edge and 6-8 points on the gel front, but the procedure could easily have been automatized.

The map between the gel image domain and the model gel domain is a transformation consisting of a dilation, a rotation and a translation. The scanning resolution and physical gel size determine the scale factor in the transformation. The slope of the top edge line gives the angle in the rotation. The vertical shift in the remaining translation is determined by the vertical position of the top edge line. However, we do not know the sideways position of the physical gel in the gel image: for example the position of the upper left corner of the physical gel along the extension of the top edge line in the image. Hence, we cannot determine the horizontal shift in the translation step in the transformation from the gel image domain to the model gel domain. We therefore introduce a horizontal translation parameter $\xi$ defined as the unknown position of the upper left corner of the gel along the top edge line. 
We parameterize the dimensionless conductivity functions with constant values $g_{l}$ and $g_{r}$, i.e. $h \gamma_{l} \equiv g_{l}$ and $h \gamma_{r} \equiv g_{r}$, one constant for each side. The partial differential equation for the dimensionless electrostatic potential is numerically solved in the PDE Toolbox in MATLAB ${ }^{\circledR}$ (MathWorks Inc., http://www.mathworks.com). A grid of points is used to specify the current leakage warping function: the area of interest $(a, b) \times(0, c)$ is partitioned into a $16 \times 16$ grid, where the grid size is chosen ad hoc. The ordinary differential equation for the warping of the 17 migration paths, one for each initial position in the partitioning of the interval $(a, b)$, is solved with a simple forward iteration after a partitioning of the $x_{2}$-dimension, and the 17 uniformly spaced points in the interval $(0, c), 17$ for each initial value, then specify the grid.

The parameters are estimated by fitting the model gel front to the image gel front. The model gel front curve is specified by three parameters: the two conductivity parameters $g_{l}$ and $g_{r}$ and the second dimension position of the gel front in the ideal gel, $c$. The image gel front curve is mapped into the model coordinate system, where the mapping needs the specification of the horizontal translation parameter, $\xi$. It is these four parameters that are estimated by a least square fitting of the two curves. This was implemented with an initial preprocessing step where the model is simulated for a discrete number of conductivity parameters, and a low-degree polynomial curves is fitted to a discrete number of gel front positions $c$. This library of curves for different $g_{l}, g_{r}$ and $c$ parameters is then searched together with the parameter space for $\xi$ to get an optimal fit of the two curves.

\subsubsection{Warping gel image}

The model is first simulated with the estimated conductivities $\hat{g}_{l}$ and $\hat{g}_{r}$. In order to include a maximum defined area in the warping of the gel image, the delimiters of the first dimension, $\hat{a}$ and $\hat{b}$, are chosen by backward iteration from the lower left and lower right corners of the gel, respectively. The grid of deformation of the scaled current leakage warping function is calculated for the area of interest $(\hat{a}, \hat{b}) \times(0, \hat{c})$ and then mapped into the gel image with the estimated translation parameter $\hat{\xi}$. The warped version of the gel image is finally obtained with Eq. (1) where bicubic spline interpolation in the algorithm interp2 in MATLAB ${ }^{\circledR}$ is used to interpolate the warping function between the grid points.

\subsection{Warping step II: Image alignment}

We automatically align the current leakage corrected images one-by-one to a reference image. For reference image we choose one of the five current leakage corrected gel images from normal growth conditions: the one with smallest maximum of the two estimated conductivity parameter. In order to use only the spot pattern in the alignment, the reference image is cropped such that all spots in the earlier analysis (Alipour et al., 2001) are present in the smaller version, while the irreproducible top part and gel front are excluded. We denote the cropped reference image with $\mu$ and let $Y$ be the image that we wish to align with $\mu$. 
First a top-hat transformation (Glasbey and Horgan, 1995) is applied to all gel images to subtract large-scale trends in the background level. The radius of the cylinder in the top-hat transformation is set to 50 pixels, far larger than any spot size but small enough to capture large-scale trends. The data in 2D gel images range over several orders of magnitude, and since we will use an intensity based similarity criterion, see Eq. (15) below, it is convenient to have the data on the same order of magnitude. The intensity in all images are therefore transformed according to

$$
\tilde{Y}_{x}=\log \left(Y_{x}+1\right), \text { for } x \in X .
$$

A tilde mark is used to denote the log-transformed versions of the images. These versions are only used in the alignment procedure and not in the final warping of the images.

\subsubsection{Penalized likelihood}

We estimate the warping function that aligns $\tilde{Y}$ with $\tilde{\mu}$ by maximizing a penalized likelihood for the warping (Glasbey and Mardia, 2001). The penalized likelihood is a functional, $P$, composed of two components:

$$
P(\tilde{Y} \mid \tilde{\mu}, f, \lambda, \mathcal{C})=L(\tilde{Y} \mid \tilde{\mu}, f)-\lambda D(f, \mathcal{C}) .
$$

The first component $L$ is the $\log$-likelihood for $\tilde{Y}$, conditional on $\tilde{\mu}$ and the warping function $f$, and measures the similarity between the warped image $\tilde{Y}_{f}$ and $\tilde{\mu}$. In the second component $D$ is a distortion criterion, chosen to be zero if $f$ belongs to $\mathcal{C}$, a null-set of functions, and $\lambda$ is a non-negative constant that gives the relative weighting between $L$ and $D$.

We use a simple Gaussian image model with independent pixel values for $\tilde{Y}$ after warping:

$$
\tilde{Y}_{f(x)} \sim \mathrm{N}\left(\tilde{\mu}_{x}, \sigma^{2}\right), \text { for } x \in X .
$$

Disregarding additive and scaling constants, the log-likelihood for $\tilde{Y}$ is then the negative sum of squared differences:

$$
L(\tilde{Y} \mid \tilde{\mu}, f)=-\frac{1}{n_{1} n_{2}} \sum_{x \in X}\left(\tilde{Y}_{f(x)}-\tilde{\mu}_{x}\right)^{2},
$$

where we have normalized with the image size because we will shift between different image resolutions in the optimization algorithm.

As null-set in the distortion criterion we choose the set of affine functions, $\mathcal{A}$, and as the distortion criterion the bending energy of thin plate splines in a finite window (Bookstein, 1989):

$$
D(f, \mathcal{A})=\sum_{i=1}^{2} \sum_{j=1}^{2} \sum_{k=1}^{2} \int_{\square}\left(\frac{\partial^{2} f_{i}}{\partial x_{j} \partial x_{k}}\right)^{2} d x,
$$

integrated over the image domain $\square=\left(0, n_{1}\right) \times\left(0, n_{2}\right)$. 


\subsubsection{Optimization algorithm}

To assess the alignment we followed the method in (Glasbey and Mardia, 2001), where the warping function is specified on a $q_{1} \times q_{2}$ grid, a piecewise bilinear function interpolates elsewhere and a conjugate gradient algorithm is used to find the maximum penalized likelihood. Since the distortion criterion in Eq. (16) involves second order derivatives, and a piecewise bilinear function is a first-degree polynomial with discontinuous first order derivatives, $D(f, \mathcal{A})$ is approximated in the method. The first partial derivatives of $P$ required by the conjugate gradient algorithm are computed using difference methods, taking advantage of the fact that the piecewise bilinear function has local support; changes in a point in the grid of deformation only affect a subset of terms in $\tilde{Y}_{f}$ and $D$.

\begin{tabular}{lrrr}
\hline Iteration & $\begin{array}{r}\text { Size of reference } \\
\text { image } \mu \text { [pixels] }\end{array}$ & Grid size $q$ & Penalty weight $\lambda$ \\
\hline 1 & $102 \times 146$ & $(1,1)$ & $30 \lambda_{0}$ \\
2 & $204 \times 292$ & $(2,3)$ & $10 \lambda_{0}$ \\
3 & $204 \times 292$ & $(4,6)$ & $3 \lambda_{0}$ \\
4 & $408 \times 584$ & $(8,12)$ & $\lambda_{0}$ \\
5 & $408 \times 584$ & $(16,24)$ & $0.3 \lambda_{0}$ \\
\hline
\end{tabular}

Table 1: The multiresolution strategy in the optimization algorithm to assess the image alignment in warping step II. The original size of the reference image $\mu$ is $816 \times 1168$ pixels.

All implementations were done in the MATLAB ${ }^{\circledR}$ software. The algorithm fminunc in the Optimization Toolbox in MATLAB ${ }^{\circledR}$ was used to maximize the penalized likelihood with respect to the $2\left(q_{1}+1\right)\left(q_{2}+1\right)$ parameters in the grid of deformation. We adopted a multi-resolution strategy to avoid being trapped in local sub-optima, see Table 1 . The strategy operates on different image resolutions, a coarse resolution in the beginning and finer scales as iterations advance, simultaneously refining the grid size $q=\left(q_{1}, q_{2}\right)$ and allowing more distortions by decreasing $\lambda$. The different image resolutions were obtained with a sequence of image reductions by $2 \times 2$ block averaging (Glasbey and Horgan, 1995), and a subsequent smoothing with a Gaussian filter. The original size of the reference image $\mu$ was $816 \times 1168$ pixels. The final grid size $q$ and the permitted distortion via the parameter $\lambda_{0}$ were chosen to maximize the number of adjacent spot pairs in the aligned images, see Section 3.2.3. 


\subsection{Evaluation}

\subsubsection{Spot displacement vectors}

We have used the list of 1028 manually matched spots from the earlier analysis of the gels (Alipour et al., 2001) to measure the geometric similarity between the spot patterns in two images. Let $\left(x_{1}^{(j)}, x_{2}^{(j)}\right)$ and $\left({ }^{r e f} x_{1}^{(j)},{ }^{r e f} x_{2}^{(j)}\right)$, where $j=1, \ldots, 1028$ is the protein identification number, denote the coordinates of the spot centers in the image $Y$ and the reference image $\mu$, respectively. The images can be either the original images, the current leakage corrected versions or the aligned versions. When superimposing the original and current leakage corrected versions, the image $Y$ is first translated such that the most dominant spot (protein Tdh3, see http://yeast-2dpage.gmm.gu.se) match. The displacement vector between the two spot centers in the spot pair that correspond to protein $j$ is given by

$$
\Delta x^{(j)}=x^{(j)}-{ }^{r e f} x^{(j)} .
$$

We define the spot pair to be an adjacent spot pair if the spot displacement is less than or equal to a mean spot radius,

$$
\text { adjacent spot pair } \Leftrightarrow\left|\Delta x^{(j)}\right| \leq \bar{\sigma} .
$$

We let the mean spot radius $\bar{\sigma}$ be the mean value of the spot half-widths (Garrels, 1989) of all spots in all ten gel images and in both directions from the earlier analysis. In our gels this radius is equal to $1.1 \mathrm{~mm}$ or equivalently 6.3 pixels.

\subsubsection{Gel image analysis in PDQuest ${ }^{\mathrm{TM}}$}

We have adopted the following strategy to create a list of scattered landmarks for the matching step in the comparison within PDQuest ${ }^{\mathrm{TM}}$ (version 6.1.1) (Bio-Rad Laboratories Inc., http://www.bio-rad.com). The reference image $\mu$ is first divided into $2 \times 3$ main rectangles. Each main rectangle is then split into $3 \times 3$ sub-rectangles. Hence there are totally 54 sub-rectangles. The most intense spot in each sub-rectangle is picked as a landmark candidate and the nine candidates in each main rectangle are ordered in descending magnitude. The full list of 54 landmarks is then obtained by starting with the most intense spot in the gel and then walking clockwise around the main rectangles, successively picking landmarks from the ordered candidate list.

The preprocessing step in PDQuest ${ }^{\mathrm{TM}}$ was done with a background subtraction with a floating ball of radius 25 pixels and a smoothing with a trimmed mean, the so called contra mean, with a $5 \times 5 \mathrm{kernel}$. Of the detection parameters the size scale was set to 5 and the sensitivity parameter was adjusted in each gel such that approximately 1250 spots were detected in a preliminary test run. Spots in the gel fronts were removed manually after the spot detection step. Landmarks were then added according to the list above and an auto-match of all gels was done after each added landmark. After all 54 landmarks had been marked, further landmarks were manually added until all spots detected in all ten gels were matched. 


\section{Results}

\subsection{Warping step I: Current leakage correction}

\subsubsection{Numerical simulation of the current leakage model}

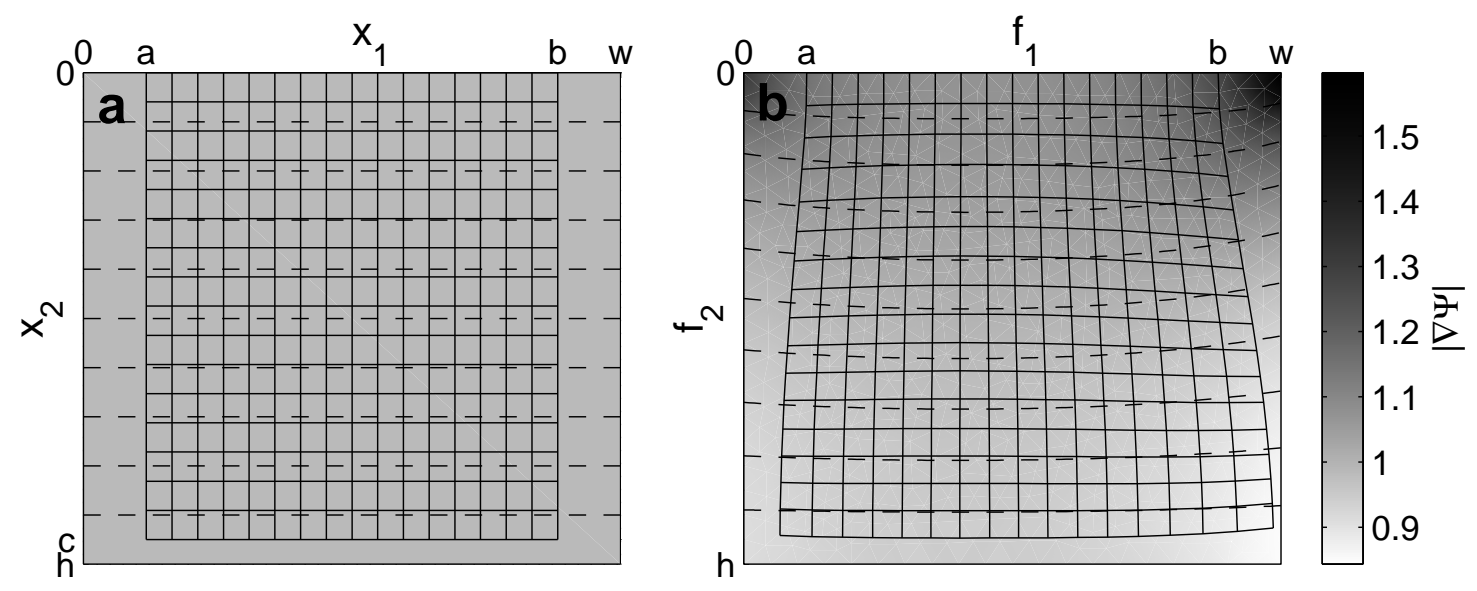

Figure 1: Warping of the protein pattern due to current leakage. (a) An ideal gel and (b) a gel with current leakage across the sides. The electric potential is visualized by equipotential lines drawn as dashed curves and the electric field strength is indicated with a grey scale intensity according to the scale shown in the bar to the right, where it is given relative to the undisturbed field strength in the ideal gel. The grid of deformation, shown with solid lines, illustrates the current leakage warping function: the map between the position $x$ a protein would have had in an ideal gel to the position $f$ in the gel with current leakage. The dimensionless conductivity parameters at the left and right boundaries are in this example $g_{l}=0.2$ and $g_{r}=0.4$, respectively.

The result of a simulation of the current leakage model is shown in Fig. 1. The dimensionless conductivity parameters are in this example set to $g_{l}=0.2$ and $g_{r}=0.4$. Hence, in this simulation we consider a case where the conductivity in the imperfect gluing of the spacer on the right hand side is twice that on the left hand side. The dashed lines show equipotential lines and the grey scale intensity corresponds to the electric field strength in terms of $V_{0} / h[\mathrm{~V} / \mathrm{m}]$ according to the gray scale in the bar to the right. The grids of deformation shown with solid lines illustrate the current leakage warping function: the map between the position $x=\left(x_{1}, x_{2}\right)$ a protein would have had in the ideal gel to the position $f(x)=\left(f_{1}(x), f_{2}(x)\right)$ it has in the gel with current leakage.

When interpreting the current leakage warping function the positions in the vertical dimension can either be seen as positions of proteins with different molecular weight at a certain time: a snapshot of the gel, or as positions of proteins with the same molecular weight but at different times: a superimposition of several snapshots. 
Studying the vertical lines in Fig. 1 (b), one can see that the SDS-protein complexes migrate in the electric field direction which slightly pulls them towards the sides of the gel. In the upper parts of the gel, the potential over the glued spacer is the largest, therefore the current leakage is the largest and the electric field deviates the most towards the sides. However, at the very top, the electric field points straight down due to the constant potential along the cathode boundary. Further down the gel, the potential is smaller and thus the leakage is also smaller, and the field reverts towards the vertical direction.

Looking at the horizontal lines in Fig. 1 (b), which indicate proteins with the same molecular weight, one can see that the inhomogeneous electric field strength in the upper part of the gel causes proteins with the same molecular weight to move faster nearer the sides than in the middle part. This lead is lost further down the gel when the electric field becomes weaker near the sides in comparison with the middle part. Finally at the very bottom, the gel front appears as a "smile" in the gel with current leakage. The curve form of this "smile" was used to estimate the parameters in the model.

\subsubsection{Current leakage corrected images}
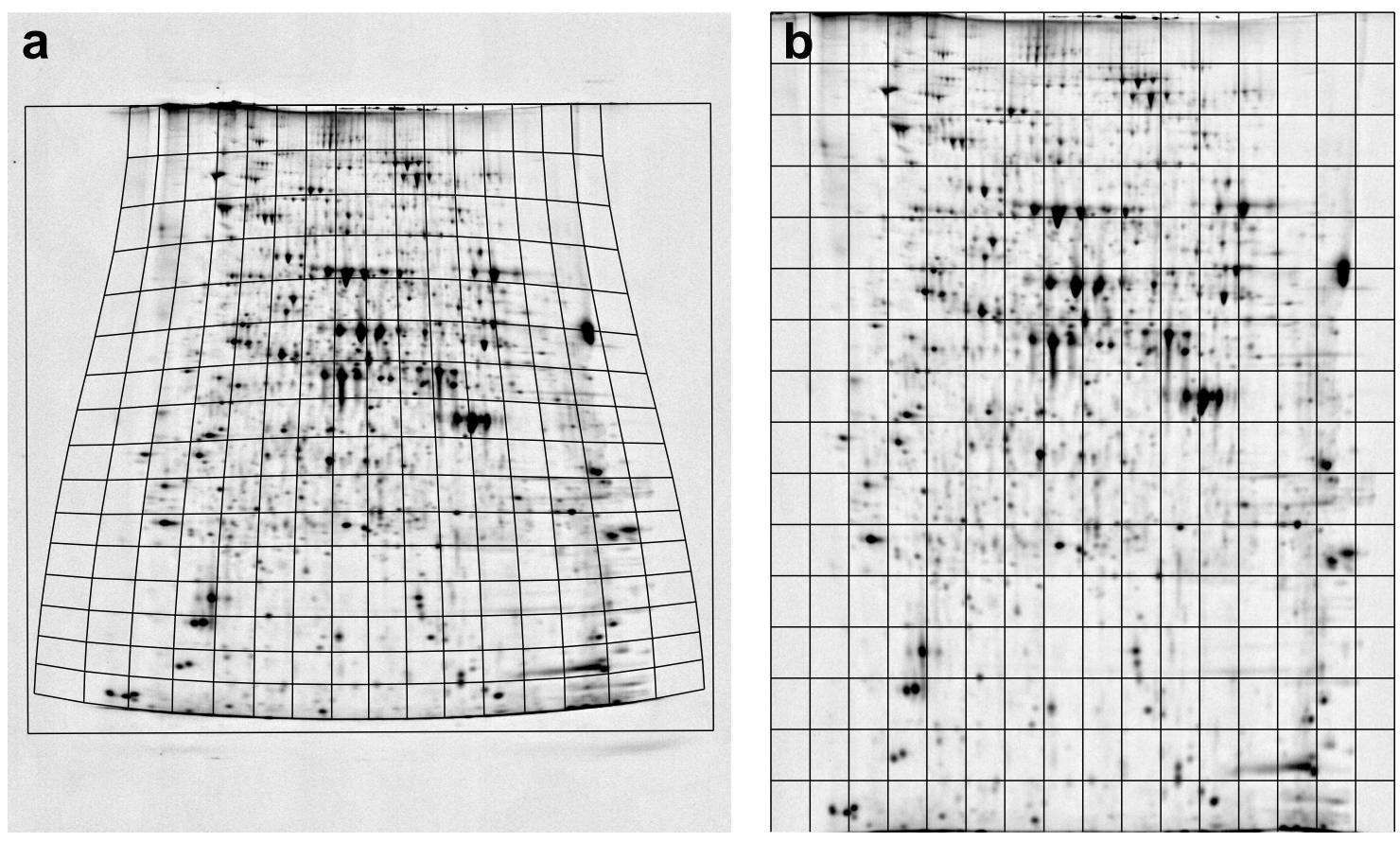

Figure 2: Warping step I: Current leakage correction of a gel image. (a) Original image (gel 1). (b) Warped and current leakage corrected version of the gel image. The grids of deformations according to the model are superimposed in the images. The estimated dimensionless conductivity parameters are $\hat{g}_{l}=0.65$ and $\hat{g}_{r}=0.7$. 
A result of the first warping step is shown in Fig. 2, where gel image 1 is warped to correct for the effect of current leakage. The dimensionless conductivity parameters for this gel were estimated to $\hat{g}_{l}=0.65$ and $\hat{g}_{r}=0.7$. The estimated parameters in the other gel images ranged between 0 and 1.175 with a mean value of 0.50 . Considering the definition of the conductivity expression in Eq. (9), this corresponds in rough numbers to that the average conductivity in the gluing of the spacers was 0.06 times the one in the gel.

\subsection{Warping step II: Image alignment}

\subsubsection{Aligned images}
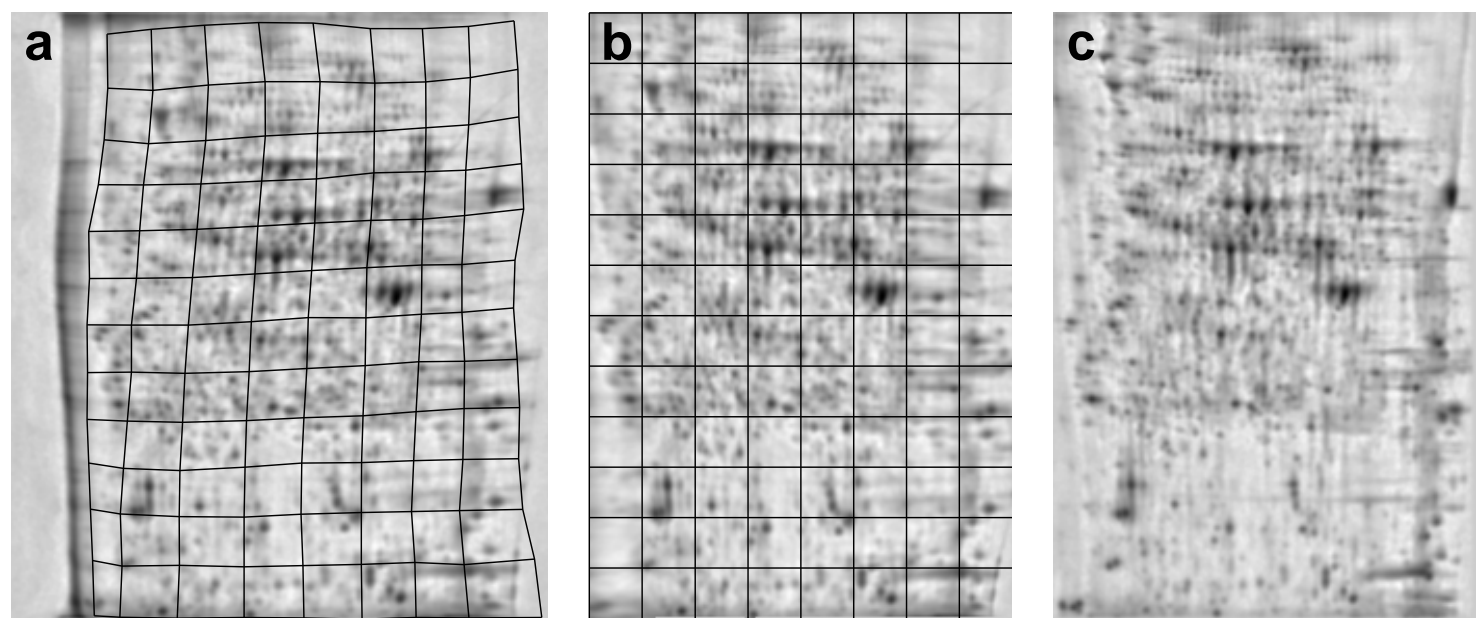

Figure 3: Warping step II: Image alignment of two current leakage corrected images. (a) Image $\tilde{Y}$ (current leakage corrected gel 6). (b) Warped version $\tilde{Y}_{f}$ to align with (c) reference image $\tilde{\mu}$ (cropped current leakage corrected gel 1). The grid of deformation for the alignment is superimposed in the images. Note the log-transformed intensities in the images.

The second warping step where the current leakage corrected images are aligned is illustrated in Fig. 3, where an alignment of the current leakage corrected gel 6 with the reference image (cropped current leakage corrected gel 1) is shown. The distortion penalty weight $\lambda_{0}$ was in this example chosen to be $10^{-3}$ and iterations were stopped after iteration four when the size of the grid of deformation was $q=(8,12)$, see Table 1 . Note the log-transformed intensities in the gel images that enhances the low-intensity parts; compare Fig. 3 (c) with Fig. 2 (b). When we tried to align the gel images without this initial intensity transformation, the algorithm primarily considered the most prominent spots due to the intensity based similarity criterion and the fact that the data in $2 \mathrm{D}$ gel images range over several orders of magnitude, and the alignment was not successful in areas with no dominant spots. 
There are two sources of variation between the two aligned gel images in Fig. 3. Firstly, they show proteins from cells grown under different conditions: image $Y$ (gel 6) from saline growth conditions and reference image $\mu$ (gel 1) from normal conditions. Secondly, the gels were run in two different electrophoresis tanks. Despite these differences the spot patterns and the reproducible background features are sufficiently similar for the alignment to succeed. However, a weakness in the intensity based similarity criterion can be seen in the lower left corner of the images. The irreproducible vertical band to the left in $Y$ is not present in $\mu$. Therefore the method avoids this area and is unable to align the spots that are embedded in this band in image $Y$.

The image alignment can also be seen in the color-superimposition in Fig. 4 (a). As in the color-superimposition of 2D gels by Horgan et al. (1992), the reference image $\mu$ is colored red and the warped image $Y_{f}$ is colored blue. When the spots in the images coincide the two complementary colors add up to grey or black. An unmatched spot pair shows up as a blue and a red spot near each other; the reason for this can either be a misaligned area, as in the lower left corner, or a protein specific change in position due to for example a phosphorylation. A single red or blue spot indicates that the protein is down-regulated or up-regulated during steady state saline growth, respectively. There are quite a number of examples of the latter in Fig. 4 (a).

\subsubsection{Spot pattern similarity}

We have used a list of 1028 manually matched spots from an earlier analysis of the gel images (Alipour et al., 2001) to measure the similarity of the spot patterns in the images. Figure 4 (b) shows the spot displacement vectors in the color-superimposition of the aligned images in Fig. 4 (a). The larger the vector is, the larger is the arrow head. Figure 4 (c) shows another representation of these displacement vectors. The vectors are translated with their starting points to the origin and their endpoints, i.e. the arrow tips, are marked with dots. The circle shows the criterion for an adjacent spot pair. The circle diameter is the mean value of the spot widths of all spots in all images. The number of dots inside the circle is the number of adjacent spot pairs. Among the largest vectors we find the misaligned spots in the lower left corner mentioned above, and horizontal displacements of alkaline proteins in the rightmost part of the gels; these proteins are sometimes hard to get properly focused in the first dimension. There are also a few number of cases where one might suspect a manual error in the earlier analysis: for example the almost vertical displacement vector in the uppermost part of Fig. 4 (b). 

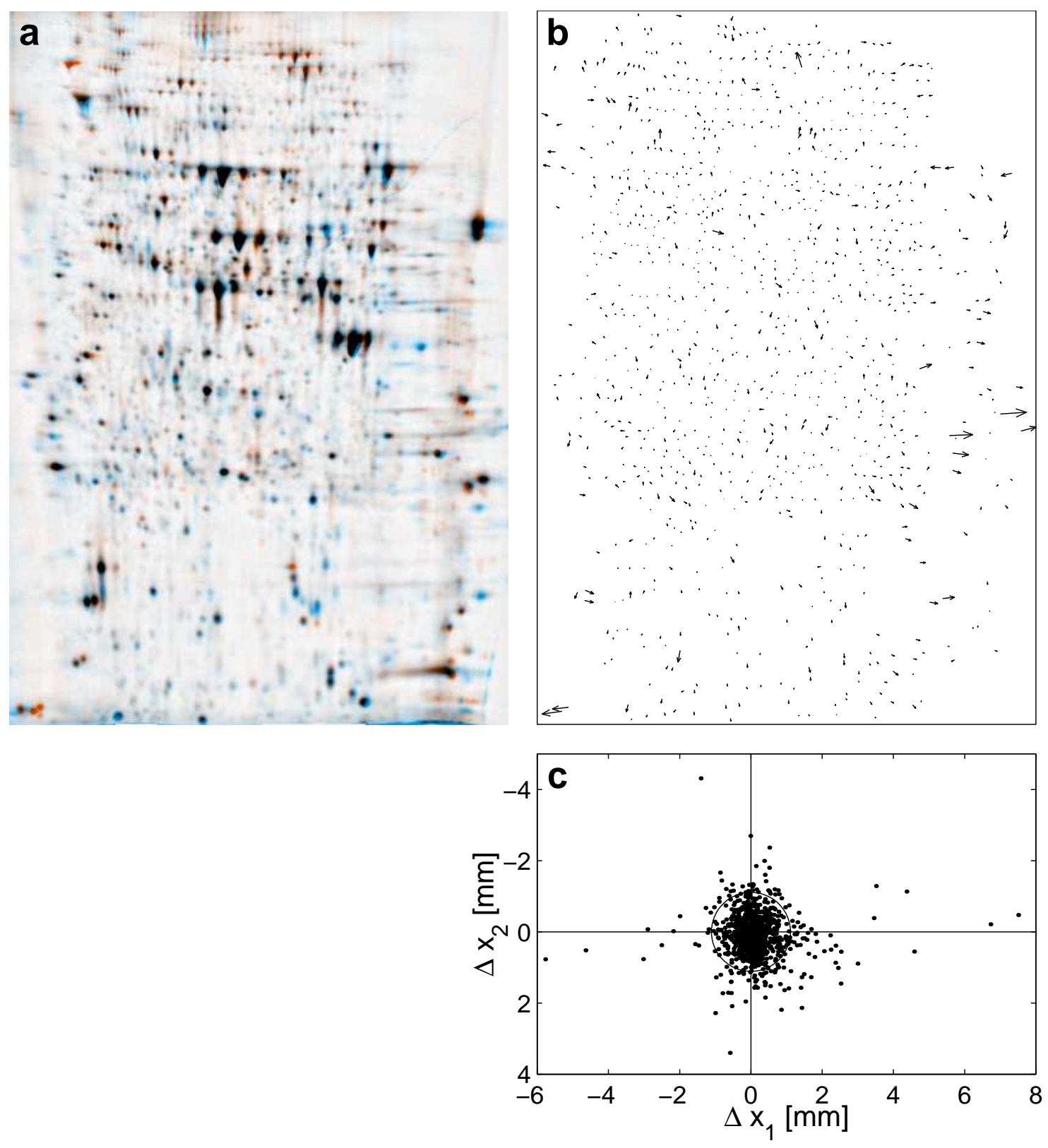

Figure 4: Similarity of the spot patterns in the aligned images in Fig. 3. (a) A colorsuperimposition of the two images: the reference image $\mu$ is colored red and the warped image $Y_{f}$ is colored blue. (b) Spot displacement vectors in the image superimposition; the larger the vector the larger the arrow head. (c) The displacement vectors are translated with their starting points to the origin and their endpoints are marked with dots. The circle shows the criterion for an adjacent spot pair. 


\subsubsection{Choice of alignment algorithm parameters}
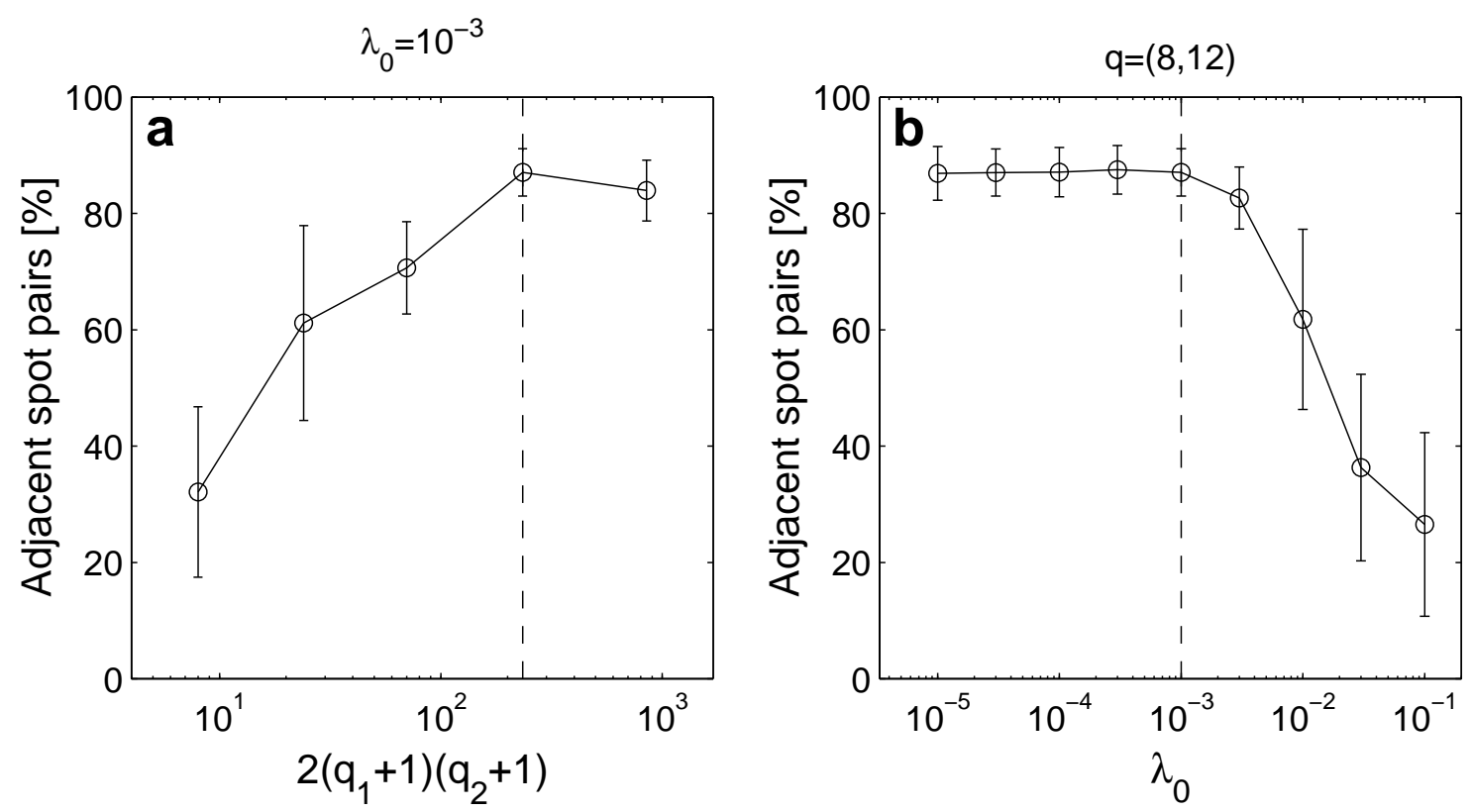

Figure 5: Spot pattern similarity in aligned images after each iteration step in the optimization algorithm and for different penalty weights. (a) The percentage of adjacent spot pairs obtained after each iteration step. Each iteration is illustrated with the number of parameters in the grid of deformation. (b) The effect of using different values of the distortion penalty weight $\lambda_{0}$ on the percentage of adjacent spots pairs. The circles mark the mean values and the error bars the standard deviations in the set of the nine images aligned to the reference image. The vertical dashed lines show the chosen final grid size and penalty weight.

Figure 5 (a) shows the successive improvement of the spot pattern similarity, measured as percentage of adjacent spot pairs, during the iterations in the alignment algorithm; the figure shows the case were the distortion penalty weight $\lambda_{0}$ was $10^{-3}$. The percentage of adjacent spot pairs is plotted as a function of the number of parameters in the successively refined grid of deformation, see Table 1 . The number of parameters in the grid is a natural choice to characterize each iteration step since it is one of the crucial properties of a multidimensional optimization problem. The circles in Fig. 5 mark the mean values and the error bars the standard deviations in the set of the nine images aligned to the reference image. The percentage of adjacent spot pairs was increased with refined grid size until a saturated value was reached after the fourth iteration step where a $8 \times 12$ grid was used; further refinement of the grid in the fifth iteration did not improve the spot pattern similarity.

Figure 5 (b) shows the effect of using different distortion penalty weights in the align- 
ment algorithm. The attained percentage of adjacent spot pairs after iteration four is plotted as a function of $\lambda_{0}$. Hard distortion penalty via large $\lambda_{0}$ corresponds to a warping function that is close to the null-set of the distortion criterion: the set of affine functions. An affine function is a combination of a dilation, a translation, a rotation and a shearing of the image. It is unable to align the images and the percentage of adjacent spot pairs was thus low for large penalty weights. The percentage was increased as more distortions were allowed until a saturated value was reached for $\lambda_{0}=10^{-3}$; further decrease of the distortion penalty weight did not improve the spot pattern similarity.

The final grid size, i.e. the number of iterations, and the distortion penalty weight for the final warping of the images were chosen to be the ones that achieved the saturated number of adjacent spot pairs, and at the same time was computationally least timeconsuming and introduced the least distortion. The choices, $\lambda_{0}=10^{-3}$ and $q=(8,12)$, are shown with vertical dashed lines in Fig. 5. The finer the grid of deformation was, i.e. the larger the parameter space for the optimization algorithm to search, the longer was the computation time. The lower the distortion penalty, the larger was the chance that the algorithm produced spurious transformations in parts of the image where there were little information for the similarity criterion. This sometime happened near the image edges for low distortion penalties (data not shown).

\subsection{Comparing the two warping steps}

The successive improvement of the spot pattern similarity after each one of the two warping steps is shown in Fig. 6. The length distribution of spot displacement vectors is plotted for the three sets of images: the original, the current leakage corrected (warping step I) and the aligned images (warping steps I+II). The current leakage correction step slightly improved the spot pattern similarity. The step had mainly corrected for large displacements in the lower part of the gel image that were caused by the wide "skirt" in the geometric distortion caused by current leakage. However, it was the second alignment step that clearly made the largest improvement.

\begin{tabular}{lrrrr}
\hline & Warping steps I+II & \multicolumn{2}{c}{ Only warping step II } \\
\hline Similarity criterion $L$ & -0.158 & $(0.085)$ & -0.151 & $(0.066)$ \\
Distortion criterion $D$ & 3.88 & $(1.61)$ & 4.80 & $(1.20)$ \\
Computation time [min] & 74 & $(16)$ & 129 & $(28)$ \\
Adjacent spot pairs [\%] & 87.1 & $(4.1)$ & 86.8 & $(4.4)$ \\
\hline
\end{tabular}

Table 2: The result of only applying the alignment step (warping step II) on the original images in comparison with the two-step warping (warping steps I+II). The numbers in the table are the means in the set of nine images and with standard deviations given in parentheses.

The result when only applying the alignment step (warping step II) on the original images in comparison to the two-step warping (warping steps I+II) is presented in Table 


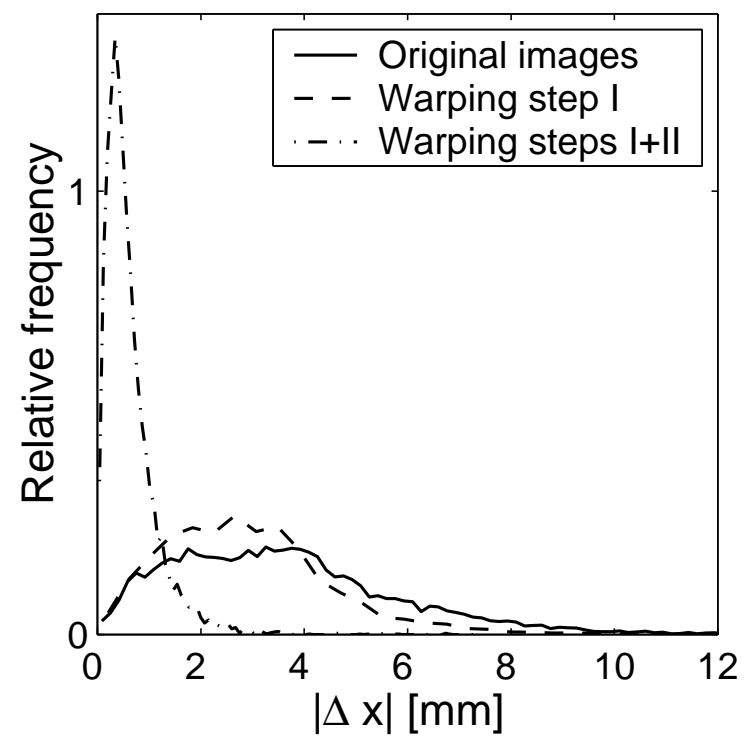

Figure 6: Comparison of the spot pattern similarity before, after one and after the two warping steps. The figure shows the length distributions of the spot displacement vectors in three sets of images: original images (solid line), current leakage corrected images (warping step I) (dashed line) and aligned images (warping steps I+II) (dash-dot line).

2. We used the same reference image, i.e. the cropped current leakage corrected gel 1 , since it did not contain the irreproducible top part and gel front. Both the image similarity measure $L$ and the spot pattern similarity measure are very much the same in the two cases. Actually, the length distribution of spot displacement vectors almost coincided with the one of the two-step warped images in Fig. 6 (data not shown). The value of the distortion criterion $D$ was slightly larger, i.e. the warping functions for alignment of the original images were further different from an affine function than the warping functions for alignment of the current leakage corrected images. The warping due to current leakage that the alignment step now also had to deal with is far different from an affine function. However, the most significant difference was in computation time: applying the alignment step on the original images instead of the current leakage corrected versions took almost twice as much computation time per gel (CPU time on a SUN Ultra 10). The first current leakage correction step merely took a minute or two per gel. 


\subsection{Comparison within PDQuest ${ }^{\mathrm{TM}}$}
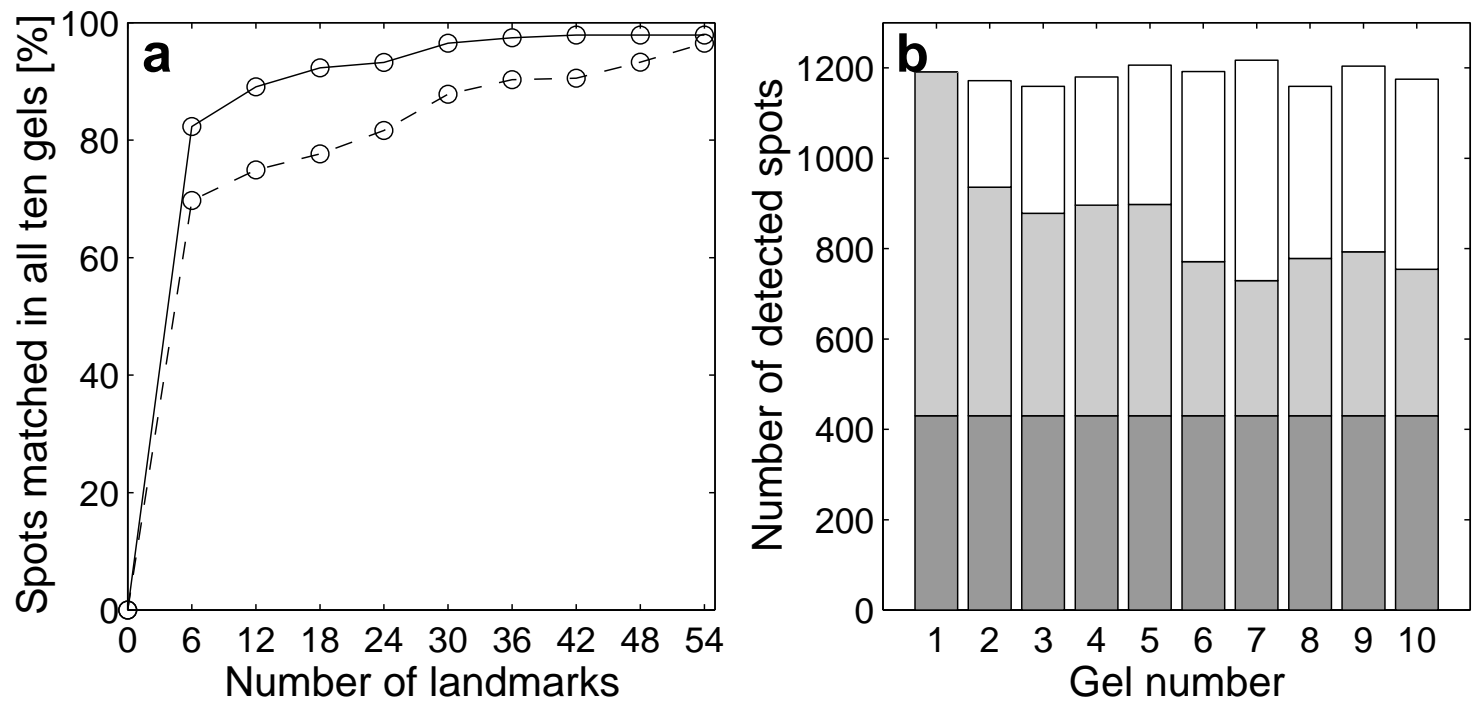

Figure 7: Gel image analysis in PDQuest ${ }^{\mathrm{TM}}$. (a) Global matching efficiencies when processing the two-step warped images (solid line) and the original images (dashed line). The number of automatically matched spots is expressed as percentage of spots detected in all ten gel images, and shown as a function of manually added landmarks. (b) Analysis of the spot detection. The bars show the number of detected spots in each gel image in the set of warped images: the spots detected in all ten images (dark gray), spots additionally in common with the reference image (gel 1) (light gray) and spots not detected in the reference image (white).

The improvement of the matching efficiency when applying the warped images to an existing matching algorithm is exemplified with the PDQuest ${ }^{\mathrm{TM}}$ software (Garrels, 1989). The global matching efficiency when processing the sets of original (dashed line) and two-step warped images (solid line) is shown in Fig. 7 (a). The number of automatically matched spots in all ten gels is plotted as a function of the number of manually added landmarks, see Section 2.4.2. The number of spots is expressed as a percentage of the number of spots that was detected in all ten gels. The matching efficiency was clearly improved when using the warped images instead of the original versions: the number of matches was larger for each number of landmarks and the total number of possible matches was reached earlier.

However, it was a limited number of spots that was detected in all ten images. Figure 7 (b) illustrates the result of the initial spot detection in the set of warped images. Although the mean number of detected spots in each gel was 1194, the number of spots that each gel had in common with the reference gel (dark + light grey) was reduced to on average 826 spots. This resulted in that there were merely 430 spots detected in all ten gels (dark grey) that could be matched and then be used in a subsequent comprehensive statistical 
analysis. The situation was similar in the set of original images (data not shown). Also note the slightly higher number of spots in common with the reference image (gel 1) within the set of gels from normal growth conditions (gels 1-5) compared with the gels from steady state saline growth (gels 6-10).

\section{Discussion}

\subsection{Current leakage correction}

The theoretical studies that model the migration behavior of SDS-protein complexes (Rodbard and Chrambach, 1970; Guo and Chen, 1990; Boileau and Slater, 2001; Locke and Trinh, 1999; Mercier et al., 2001) aim at deriving a relation between the size of a protein and its mobility coefficient. They all consider the migration of the SDS-protein complexes in the gel fiber net at a microscopic scale. Most studies assume a constant electric field, whereas Locke and Trinh (1999) and Mercier et al. (2001) investigate curved electric field lines at this size scale. We have worked on a macroscopic scale to investigate the effect that an inhomogeneous global electric field caused by current leakage has on the protein spot pattern. Since we are interested in relative position changes due to the altered global electric field, the mobility coefficient cancels out in the differential equation (10) for the warping function.

The warping function produced by the current leakage model seems similar to the warping function in the method to correct for "frowns" and "smiles" in multi-track electrophoresis gels proposed by Glasbey and Wright (1994). They also treat the images individually, but they can use the fact that bands in these gels are asymmetric to estimate orientation directions and to mathematically derive a warping function of the whole image. We have merely used information in the top and bottom part of the gel images and have had to rely on the physicochemical model in between.

When evaluating the result of the current leakage correction step in Fig. 6, one should have the following in mind: the images are treated individually with no knowledge of the spot patterns in the other gels and the choice of a constant functions to parameterize the unknown conductivity function along the side is clearly too simple; the imperfections in the gluing of the spacers are likely to be more heterogeneous.

\subsection{Automatic image alignment}

We agree with Smilansky (2001) that spot pattern features, such as spot shapes, streaks and smears, and background structures are reproducible and can be used by pixel-based methods to align the protein spot pattern in gel images. The image warping technique for automatic alignment of images has been used extensively in the field of digital image analysis (Glasbey and Mardia, 1998). With the exception of an early example by Conradsen and Pedersen (1992), the technique seems not to have been applied to 2D gels until very recently (Smilansky, 2001). We find that the penalized likelihood formulation proposed 
by Glasbey and Mardia (Glasbey and Mardia, 2001) is a powerful tool for the automatic alignment of gel images.

Our similarity criterion - the log-likelihood based on a Gaussian image model for the images after warping - is the well known negative sums of squared differences in machine vision literature. This criterion was also used by Conradsen and Pedersen (1992). However, we applied an initial log-transformation of the intensities in the images. This essentially corresponds to that we have used a log-normal image model for the images after warping without an initial log-transformation. The similarity measure used by Smilansky (2001) is described in general terms and consists of several components based on both contrast and gradient information in the images.

The largest conceptual difference of our method compared with the earlier warping methods (Conradsen and Pedersen, 1992; Smilansky, 2001) is that we keep explicit control of the distortions introduced in the warping steps: in the first warping step the distortion is a smooth function derived from a physicochemical model, and in the second alignment step rough distortions are penalized by a criterion consisting of the bending energy of thin-plate splines (Bookstein, 1989) which is minimized by the set of affine functions. Conradsen and Pedersen (1992) do not consider any distortion penalty at all and the assessed warping function that aligns their gel images seems rough. Smilansky (2001) keeps implicit control of the introduced distortions by restrictions in the iteration loop. Given an undistorted reference gel, the value of our distortion criterion can serve as a measure of the geometric distortion of the spot pattern in the gel image that is aligned with the reference.

There is also a difference in computation time between the method proposed by Smilansky (2001), which is based on a large number of alignments of small sub-images, and the methods proposed by Conradsen and Pedersen (1992) and us, which both apply an alignment of the whole image. Smilansky's method is much faster in computation time, seconds or minutes per gel, while our implementation took about an hour per gel. However, our method seems robust; there was no need for any manual interference to guide the alignment as sometimes is the case in Smilansky's faster method.

From the comparison when the alignment step was applied on the original images instead of the two-step procedure, see Table 2, we can conclude that the final result is essentially the same but that the current leakage correction step clearly gives the automatic alignment step more similar images and consequently a shorter computation time. When analyzing gels where there have been no current leakage during the second dimension electrophoresis and therefore less geometric distortions, one can probably skip the first current leakage correction step.

Given an undistorted reference gel image, a next step might be to combine the two warping steps into one single alignment step by formulating a distortion criterion that has as null-set the functions that satisfy the differential equations in the physicochemical model. 


\subsection{Image warping methods in $2 \mathrm{D}$ gel image analysis}

The geometric distortions of the spot pattern seems smooth, which makes them correctable, e.g. by using piecewise low-degree polynomials on relatively sparse grids. This deduction is supported by the result when a piecewise bilinear function was used in the image alignment and the effect of refining the grid and allowing more distortions was examined: a saturated number of adjacent spot pairs was reached, see Fig. 5, and most of the remaining pattern dissimilarities seem to be spot-specific displacements relative to the overall spot pattern, see Fig. 4 (b). We therefore conclude that one can divide the spot matching problem into two separate parts: a primary global correction for smooth non-spot-specific distortions where one regards the whole image, and a second more traditional spot-specific matching step where one can use a feature-based or pixel-based matching algorithm.

Voss and Haberl (2000) pointed out two major problems that push matching algorithm to their limit and have consequences for a comprehensive statistical analysis: geometric distortions of the spot patterns and irreproducibilities in spot intensities. In their study of a set of 49 gels, the global matching efficiency seems hampered primarily because of these two problems and only a limited number of spots is matched in all gels. Although we think that we have corrected for smooth geometric distortions, we see a similar limited number of matched spots in all gels in Fig. 7 (b). The reason for this is problems with the crucial spot detection step; there is a large number of spots that are near the detection threshold and because the images are treated individually, spots corresponding to the same protein are above this threshold in some images and below in other.

Despite the fact that the warping technique proposed in this article is computationally time-consuming we think one should weigh, especially in the case of large-scale proteomics, this cost against the fact that the method is automatic and seems to be robust.

With the implementations in the present study it is not possible to quantify the spot contents in the warped images. The image warping as defined in Eq. (1) does change the protein spot content in the images (data not shown). Mathematically, a change in spot contents can be avoided by compensating for the area scale in the warping function and using a slightly different definition of the warped image compared with the one in Eq. (1): it reads

$$
\left(Y_{f}\right)_{x}=Y_{f(x)}\left|\frac{\partial f}{\partial x}\right| \text { for } x \in X,
$$

where the area scale $|\partial f / \partial x|$ is the Jacobian determinant of the warping function. We have not implemented this area scale compensation in this study since we in the second warping step have used a piecewise bilinear function that has discontinuous first order derivatives. A solution might be to use bicubic B-splines instead: a piecewise third-degree polynomial that has continuous second order derivatives and a local support (Rueckert et al., 1999). In the present study we have started by considering the use of one of the simplest numerical approximations of arbitrary functions: the piecewise bilinear function, and have not taken a step to the B-spline in the hierarchy of function complexity. 


\section{Acknowledgments}

The authors have been supported by Chalmers Bioinformatics Programme, Stochastic Centre in Göteborg and the Swedish Research Council. We are also indebted to Chris Glasbey for valuable discussions.

\section{References}

Alipour, H., Farea, T., Norbeck, J., Ericson, E., Mostad, P., Nerman, O., Brown, P., Botstein, D. and Blomberg, A. (2001). Genome wide analysis of expression changes in Saccharomyces cereviviae during steady-state saline growth. Manuscript.

Appel, R. D., Vargas, J. R., Palagi, P. M., Walther, D. and Hochstrasser, D. F. (1997). MELANIE II - a third generation software package for analysis of two-dimensional electrophoresis images: II. Algorithms, Electrophoresis 18: 2735-2748.

Bier, M., Palusinski, O. A., Mosher, R. A. and Saville, D. A. (1983). Electrophoresis: Mathematical modeling and computer simulation, Science 219: 1281-1287.

Blomberg, A., Blomberg, L., Norbeck, J., Fey, S., Larsen, P., Larsen, M., Roepstorff, P., Degand, H., Boutry, M., Posch, A. and Görg, A. (1995). Interlaboratory reproducibility of yeast protein patterns analyzed by immobilized pHgradient two-dimensional gel electrophoresis, Electrophoresis 16: 1935-1945.

Boileau, J. and Slater, G. (2001). An exactly solvable Ogston model of gel electrophoresis. VI. Towards a theory for macromolecules., Electrophoresis 22: 673-683.

Bookstein, F. L. (1989). Principal warps: Thin-plate splines and the decomposition of deformations, IEEE Transactions on Pattern Analysis and Machine Intelligence 11: 567585 .

Conradsen, K. and Pedersen, J. (1992). Analysis of two-dimensional electrophoretic gels, Biometrics 48: 1273-1287.

Garrels, J. I. (1989). The QUEST system for quantitative analysis of two-dimensional gels, Journal of Biological Chemistry 264: 5269-5289.

Glasbey, C. A. and Horgan, G. W. (1995). Image Analysis for the Biological Sciences, Wiley, Chichester.

Glasbey, C. A. and Mardia, K. V. (1998). A review of image-warping methods, Journal of Applied Statistics 25: 155-171.

Glasbey, C. A. and Mardia, K. V. (2001). A penalized likelihood approach to image warping, Journal of the Royal Statistical Society, Series B 63: 465-514. 
Glasbey, C. A. and Wright, F. (1994). An algorithm for unwarping multitrack electrophoretic gels, Electrophoresis 15: 143-148.

Guo, X. H. and Chen, S. H. (1990). The structure and thermodynamics of protein-SDS complexes in solution and the mechanism of their transport in gel-electrophoresis process, Chemical Physics 149: 129-139.

Horgan, G., Creasey, A. and Fenton, B. (1992). Superimposing two-dimensional gels to study genetic variation in malaria parasites, Electrophoresis 13: 871-875.

Kozulic, B. (1995). Models of gel electrophoresis, Analytical Biochemistry 231: 1-12.

Lemkin, P. E. (1997). Comparing two-dimensional electrophoretic gel images across the Internet, Electrophoresis 18: 461-470.

Locke, B. R. (1998). Electrophoretic transport in porous media: A volume-averaging approach, Industrial and Engineering Chemistry Research 37: 616-625.

Locke, B. R. and Trinh, S. H. (1999). When can the Ogston-Morris-Rodbard-Chrambach model be applied to gel electrophoresis?, Electrophoresis 20: 3331-3334.

Mercier, J.-F., Tessier, F. and Slater, G. W. (2001). An exactly solvable Ogston model of gel electrophoresis: VIII. Nonconducting gel fibers, curved field lines, and the NernstEinstein relation, Electrophoresis 22: 2631-2638.

Norbeck, J. and Blomberg, A. (1997). Metabolic changes associated with growth of Saccharomyces cerevisiae in $1.4 \mathrm{M}$ sodium chloride: Evidence for osmotic introduction of glycerol dissimilation via dihydroxyacetone pathway, Journal of Biological Chemistry 272: $5544-5554$.

Pleissner, K.-P., Oswald, H. and Wegner, S. (2001). Proteomics, BIOS Scientific Publishers, Oxford, chapter Image analysis of two-dimensional gels, pp. 131-149.

Pleissner, K.-P., Sander, S., Oswald, H., Regitz-Zagrosek, V. and Fleck, E. (1997). Towards design and comparison of World Wide Web-accessible myocardial two-dimensional gel electrophoresis protein databases, Electrophoresis 18: 480-483.

Rodbard, D. and Chrambach, A. (1970). Unified theory of gel electrophoresis and gel filtration, Proceedings of the National Academy of Sciences of the USA 65: 970-977.

Rueckert, D., Sonoda, L. I., Hayes, C., Hill, D. L., Leach, M. O. and Hawkes, D. J. (1999). Nonrigid registration using free-form deformations: application to breast MR images, IEEE transactions on Medical Imaging 18: 712-721.

Smilansky, Z. (2001). Automatic registration for images of two-dimensional protein gels, Electrophoresis 22: 1616-1626. 
Voss, T. and Haberl, P. (2000). Observations on the reproducibility and matching efficiency of two-dimensional electrophoresis gels: Consequences for comprehensive data analysis, Electrophoresis 21: 3345-3350.

Westerhuis, W. H. J., Sturgis, M. N. and Niederman, R. A. (2000). Reevaluation of the electrophoretic migration behavior of soluble globular proteins in the native and detergent denatured states in polyacrylamide gels, Analytical Biochemistry 284: 143-152. 